\title{
The Diversity Study of Asteraceae Family As Effort to Develop Ecotourism in Mount Lawu
}

\author{
Sunarto \\ Department of Biology \\ Faculty of Mathematics and Natural Sciences \\ Sebelas Maret University, Surakarta. Indonesia \\ Corresponding E-mail: rm.sunarto@yahoo.com \\ Titik Warsiti \\ Department of Biology \\ Faculty of Mathematics and Natural Sciences \\ Sebelas Maret University, Surakarta. Indonesia \\ Sugiyarto \\ Department of Biology \\ Faculty of Mathematics and Natural Sciences \\ Sebelas Maret University, Surakarta. Indonesia \\ Widhi Himawan \\ Department of Biology \\ Faculty of Mathematics and Natural Sciences \\ Sebelas Maret University, Surakarta. Indonesia
}

\begin{abstract}
Asteraceae Family is a one of vegetation elements covered Mount Lawu forest area. The study purpose to determine the diversity level, abundance and distribution patterns of Asteraceae Family. This research also try to formulate the potential of Asteraceae Family as effort to develop an ecotourism of the Cemoro Kandang hiking track on Mount Lawu. The methods of this research is exploration, interview, and questionnaire. The data analyzed by descriptive quantitative method with calculation of diversity indices (Shannon-Weiner Index), density, frequency, importance value index (IVI) and distribution analysis. This research also utilizing correlation and regression tests with assistance of SPSS application. This research observed 15 species namely : Adenostema hirsutum L., Anaphalis javanica (Bl.) Boerl., Anaphalis longifolia (Bl.) DC., Chromolaena odorata L., Crassocephalum crepidioides (Benth.) S.Moore.,Dichrocephala chrysanthemifolia (B1.) DC., Emilia sonchifolia L., Erigeron karviskianus L., Eupatorium riparium Reg., Lactuca serriola L., Mikania micrantha L., Shoncus arvensis L.,Taraxacum offinale L., Tithonia diversifolia L., and Youngia japonica $\mathrm{L}$. The plants diversity levels of Asteraceae in Cemoro Kandang hiking track included in medium category. The abundance of Asteraceae was mostly found in station $V$ which located between 2600-2800 meters above sea level (masl) consisting of 41 individuals and 7 species. The lowest diversity observed in station II which located between 2000-2200 masl. consisting of 15 individuals and 1 species. Distribution patterns of Asteraceae along Cemoro Kandang hiking track were in clumped and uniform patterns. The Asteraceae have a potential as an effort to develop ecotourism through local wisdom values including natural tourism potential $(19 \%)$, education potential
\end{abstract}

(20\%), conservation potential (19\%), economy potential (19\%) and spiritual potential (23\%).

Keywords-Asteraceae, ecotourism, mount lawu, diversity

\section{INTRODUCTION}

The local wisdom values of Mount Lawu be represented by flora-fauna biodiversity, beautiful natural scenery, cultural objects, history and local people living patterns. This potential is in line with vision of ecotourism development. The vision, according to Fandeli (2000) is conservation of biodiversity and also the ecosystem through community development. Changes of forest ecosystem as effect of anthropogenic activities and natural disasters (i.e: landslide and forest fires) allows an addition or substraction of plant biodiversity (Maisyaroh, 2010). Mount Lawu ecotourism development research are conducted rarely before. Intensive activities to supports forest sustainability (likes bio-inventory) should be done as effort of ecotourism development.

Asteraceae family is a part of plant biodiversity on Mount Lawu. This plant family is a part of forest floor covering vegetation. Some of the species have a natural tourism attraction for example Edelweis (Anaphalis javanica) flowers (Kayowuan, 2014). The pharmacology industries used Asteraceae plants as medicine raw material recently. The content of biochemical compounds inside should be a cure for many disease (Subhashini and Pooguzhali, 2014). 
Cemoro Kandang is a one of hiking trails to the peak of the Mount Lawu (namely Hargo Dumilah). This site had attracts many local climbers and tourist because of natural beauty condition surroundings. Natural forest condition has not been researched numerously before, especially for Asteracea family. Therefore, the research of biodiversity and potential of the Asteracea family have an important value as efforts to develop and improve ecotourism attraction of Cemoro Kandang.

\section{METHOD}

\section{A. Research location and period}

This research conducted in December 2015 until February 2016 on Cemoro Kandang hiking trails, an ecotourism area of Mount Lawu, Karanganyar, Central Java.

\section{B. Instruments and materials}

Research instruments used in data collection including data sheet, stationary, thermometer, altimeter, global positioning system. Luxmeter, hygrometer, anemometer, $\mathrm{pH}$ meter, roll meters/raffia ropes, camera and other mountain climbing equipment. An instruments and material used to produce herbarium including stationary, portable knife, plastic, scissor, sasak (woven bamboo), oven, cardboards, binder/cellophane tape, needle, yarn and manila paper. The species identification had been done by utilizing identification books likes "Flora"(Steenis, 1987) and "Flora of Java Mountain" (Steenis, 2010).

\section{Research procedures}

The sampling station determined by altitude differences on Cemoro Kandang-Hargo Dumilah track. Each of them are station I (1800< A <2000 masl), station II (2000< A $<2200$ masl), station III (2200< A $<2400$ masl), station IV (2400< A $<2600$ masl), station V (2600< A <2800 masl), station VI (2800< A <3000 masl) and station VII (3000< A <3200 masl). Vegetation analysis in each station utilized quadrant method with size of 1x1 m2 (Odum, 1993).

\section{FINDING AND DISCUSSION}

\section{A. The Diversity Of Asteraceae Family}

Shannon Weiner Diversity Index calculation resulted value of $\mathrm{H}^{\prime}<1$, precisely in range of 0 (lowest) - 0,68 (highest). This condition as effect of different growth adaptive ability in plants on various alititude. Diversity index of station II $(2000 \leq$ A <2200 masl) determined zero (0) value in diversity. Only one spesies was found in this station, it was Eupatorium riparium Reg. Station II dominating by homogen forest contains of weeds. It makes the competition for nutrient and climate factors became very high. Observe of station $\mathrm{V}$ (2600 $\leq$ A $<2800$ masl) determined a diversity index of 0,68 consists of 7 species. This amount of Asteraceae species is the highest among all stations. Station V is not tree sheltered area as impact of forest-fires.

Generally, The Shannon Weiner diversity index (H') represented Cemoro Kandang hiking trails is 1,14. This value classified a medium diversity for Asteraceae Family existence in Cemoro Kandang. Factors influenced biodiversity of an Asteraceae Family in Cemoro Kandang is species distribution, dominancy, limiting factors, competition and presence of predators. Furthermore, the biodiversity of Cemoro Kandang also affected by forest fires that recently took place. Forest fires automatically and rapidly decreased the diversity numbers of various plants including Asteraceae Family. TABLE 1. INVENTORY ReSUlt OF ASTERACEAE FAMILY IN CEMORO KANDANG HiKing TRAILS OF MOUNT LAWU

\begin{tabular}{|c|c|c|c|c|}
\hline No. & Scientific name & Local name & $\begin{array}{c}\text { Sum of } \\
\text { individu }\end{array}$ & $\begin{array}{c}\text { Plants } \\
\text { Morphology }\end{array}$ \\
\hline 1. & Adenostema hirsutum $\mathrm{L}$. & - & 2 & Herb \\
\hline 2. & Anaphalis javanica (B1.) Boerl. & Edelweis & 19 & Shrub \\
\hline 3. & Anaphalis longifolia (B1.) DC. & Edelweis & 2 & Shrub \\
\hline 4. & Chromolaena odorata $\mathrm{L}$. & Kirinyu & 17 & Shrub \\
\hline 5. & $\begin{array}{l}\text { Crassocephalum crepidioides (Benth.) } \\
\text { S.Moore }\end{array}$ & Junggul & 7 & Herb \\
\hline 6. & Dichrocephala chrysanthemifolia (B1.) DC. & Jotang & 4 & Herb \\
\hline 7. & Emilia sonchifolia $\mathrm{L}$. & Tempuh wiyang & 6 & Herb \\
\hline 8. & Erigeron karviskianus L. & Kembang pikok & 8 & Herb \\
\hline 9. & Eupatorium riparium $\mathrm{Reg}$. & Tekelan & 85 & Herb \\
\hline 10. & Lactuca serriola $\mathrm{L}$. & Selada berduri & 1 & Herb \\
\hline 11. & Mikania micrantha $\mathrm{L}$. & Sembung Rambat & 2 & Herb \\
\hline 12. & Shoncus arvensis L. & Tempuyung & 1 & Herb \\
\hline 13. & Taraxacum offinale $L$. & Jombang & 6 & Shrub \\
\hline 14. & Tithonia diversifolia $\mathrm{L}$. & Kembang bulan & 5 & Herb \\
\hline 15. & Youngia japonica L. & Jukut & 7 & Herb \\
\hline
\end{tabular}


TABLE 2. Diversity INDEX of Asteraceae Family by Various Altitude on Cemoro Kandang Hiking Trails of Mount LaWu

\begin{tabular}{|c|c|c|c|}
\hline Station & Altitude (masl) & Scientific name & $\begin{array}{c}\text { Indeks } \\
\text { Keanekaragaman } \\
\end{array}$ \\
\hline I & $1.800 \leq \mathrm{K}<2.000$ & $\begin{array}{l}\text { a. Crassocephalum crepidioides (Benth.) } \\
\text { S.Moore } \\
\text { b. Mikania micrantha } \mathrm{L} . \\
\text { c. Taraxacum offinale L. } \\
\text { d. Erigeron karviskianus } \mathrm{L} \text {. } \\
\text { e. Chromolaena odorata } \mathrm{L} . \\
\text { f. Eupatorium riparium } \mathrm{Reg} \text {. }\end{array}$ & 0,66 \\
\hline II & $2.000 \leq \mathrm{K}<2.200$ & a. Eupatorium riparium Reg. & 0 \\
\hline III & $2.200 \leq \mathrm{K}<2.400$ & $\begin{array}{l}\text { a. Anaphalis javanica (Bl.) Boerl. } \\
\text { b. Eupatorium riparium Reg. }\end{array}$ & 0,06 \\
\hline IV & $2.400 \leq \mathrm{K}<2.600$ & $\begin{array}{l}\text { a. Chromolaena odorata } \mathrm{L} . \\
\text { b. Adenostema hirsutum } \mathrm{L} . \\
\text { c. Eupatorium riparium } \mathrm{Reg} .\end{array}$ & 0,40 \\
\hline $\mathrm{V}$ & $2.600 \leq \mathrm{K}<2.800$ & $\begin{array}{l}\text { a. Anaphalis javanica (Bl.) Boerl. } \\
\text { b. Taraxacum offinale L. } \\
\text { c. Youngia japonica } \mathrm{L} \text {. } \\
\text { d. Tithonia diversifolia } \mathrm{L} . \\
\text { e. Eupatorium riparium Reg. } \\
\text { f. Emilia sonchifolia } \mathrm{L} \text {. } \\
\text { g. Shoncus arvensis } \mathrm{L} \text {. }\end{array}$ & 0,68 \\
\hline VI & $2.800 \leq \mathrm{K}<3.000$ & $\begin{array}{l}\text { a. Anaphalis javanica }(\mathrm{Bl} .) \text { Boerl. } \\
\text { b. Taraxacum offinale L. } \\
\text { c.Dichrocephala chrysanthemifolia (B1.) DC. } \\
\text { d. Erigeron karviskianus } \mathrm{L} . \\
\text { e. Eupatorium riparium } \mathrm{Reg} .\end{array}$ & 0,50 \\
\hline VII & $3.000 \leq \mathrm{K}<3.200$ & $\begin{array}{l}\text { a. Anaphalis javanica (B1.) Boerl. } \\
\text { b. Anaphalis longifolia (B1.) DC. } \\
\text { c.Dichrocephala chrysanthemifolia (B1.) DC. } \\
\text { d. Erigeron karviskianus L. } \\
\text { e. Lactuca serriola } \mathrm{L} \text {. } \\
\text { f. Emilia sonchifolia } \mathrm{L} \text {. }\end{array}$ & 0,48 \\
\hline \multicolumn{3}{|c|}{ Diversity Index of Asteracea Family } & 1,14 \\
\hline
\end{tabular}


TABLE 3. Density Values, Relative Densities, Frequencies, Relatives Frequencies And Importance Value Index (Ivi) OF Asteraceae Family

\begin{tabular}{|c|c|c|c|c|c|c|}
\hline No & Scientific name & $\begin{array}{c}\text { Density } \\
\text { (individu/m²) }\end{array}$ & $\begin{array}{c}\text { Relative } \\
\text { density }(\%)\end{array}$ & $\begin{array}{l}\text { Frequencies } \\
\text { (plots) }\end{array}$ & $\begin{array}{c}\text { Relative } \\
\text { frequency }(\%)\end{array}$ & IVI $(\%)$ \\
\hline 1 & Adenostema hirsutum $\mathrm{L}$. & 0,678 & 11,046 & 0,142 & 13,333 & 24,379 \\
\hline 2 & Anaphalis javanica (B1.) Boerl. & 0,071 & 1,162 & 0,035 & 3,333 & 4,496 \\
\hline 3 & Anaphalis longifolia (B1.) D.C. & 0,25 & 4,069 & 0,035 & 3,333 & 7,403 \\
\hline 4 & Chromolaena odorata $\mathrm{L}$. & 0,071 & 1,162 & 0,035 & 3,333 & 4,496 \\
\hline 5 & $\begin{array}{ll}\text { Crassocephalum } & \text { crepidioides } \\
\text { (Benth.) S.Moore }\end{array}$ & 0,214 & 3,488 & 0,107 & 10 & 13,488 \\
\hline 6 & $\begin{array}{l}\text { Dichrocephala } \\
\text { chrysanthemifolia (B1.) DC. }\end{array}$ & 0,142 & 2,325 & 0,071 & 6,666 & 8,992 \\
\hline 7 & Emilia sonchifolia $\mathrm{L}$. & 0,25 & 4,069 & 0,035 & 3,333 & 7,403 \\
\hline 8 & Erigeron karviskianus L. & 0,178 & 2,906 & 0,035 & 3,333 & 6,240 \\
\hline 9 & Eupatorium riparium Reg. & 0,285 & 4,651 & 0,107 & 10 & 14,651 \\
\hline 10 & Lactuca serriola $\mathrm{L}$. & 0,607 & 9,883 & 0,071 & 6,666 & 16,550 \\
\hline 11 & Mikania micrantha $\mathrm{L}$. & 0,035 & 0,581 & 0,035 & 3,333 & 3,914 \\
\hline 12 & Shoncus arvensis L. & 0,071 & 1,162 & 0,035 & 3,333 & 4,496 \\
\hline 13 & Taraxacum offinale $\mathrm{L}$. & 3,035 & 49,418 & 0,214 & 20 & 69,418 \\
\hline 14 & Tithonia diversifolia $\mathrm{L}$. & 0,214 & 3,488 & 0,071 & 6,666 & 10,155 \\
\hline 15 & Youngia japonica $\mathrm{L}$. & 0,035 & 0,581 & 0,035 & 3,333 & 3,914 \\
\hline & Sum total & 6,142 & 100 & 1,071 & 100 & 200 \\
\hline
\end{tabular}

The highest importance value index (IVI) was obtain in species of Eupatorium riparium $(69,418 \%)$. The lowest IVI is obtain in Lactuca serriola, L. and Shoncus arvensis, L. $(3,914 \%)$. Species with highest IVI can be used to determine the vegetation type of the area. According to the IVI, Eupatorium riparium is the highest numbers of species found in all stations. The Eupatorium riparium is more often found in altitude of $2600<$ A $<2800$ masl and automatically also have highest individual number than other altitudes. Eupatorium riparium classified as herbs which usually roled as forest floor coverings plant.

Abundance and distribution of Asteraceae Family

\section{Abundance}

Individuals abundance of Asteraceae species mostly found in station $\mathrm{V}$ which located in altitude of $2600<\mathrm{A}<2800$ masl. Research of this station found 41 individuals classified in 7 species. The lowest abundance is in station II $(2000<\mathrm{A}<2200$ masl) which observed 15 individuals classified in only 1 species. The abundance of Asteraceae Family in Cemoro Kandang determined a value of 1,14 , classified as medium abundances criteria. This abundance affected by adaptation ability and life resources availability of each different species

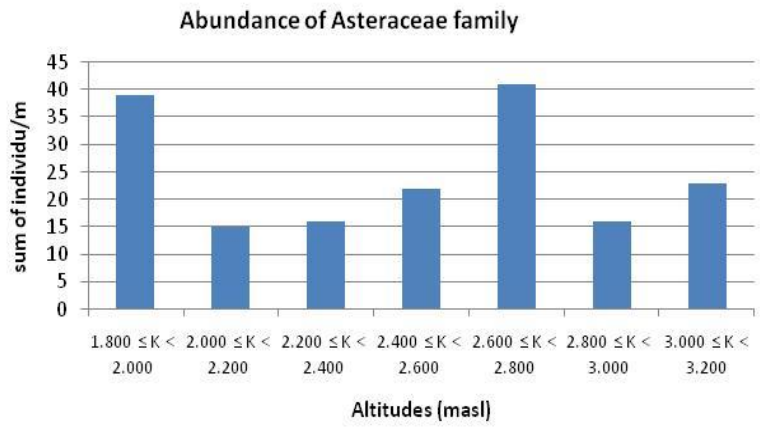

Fig. 1. Abundance of Asteraceae Family in 7 Research Stations of Cemoro Kandang Hiking Trails 


\section{Distribution pattern}

TABLE 4. DistribUtion PATTERnS OF ASTERACEAE FAMILY ON CEMORO KANDANG HIKING TRAILS

\begin{tabular}{|c|c|c|c|}
\hline No & Scientific name & $\begin{array}{l}\text { Morista } \\
\text { Indeces } \\
\end{array}$ & $\begin{array}{c}\text { Distribution } \\
\text { patterns }\end{array}$ \\
\hline 1 & Adenostema hirsutum L. & 1,5 & Clumped \\
\hline 2 & $\begin{array}{l}\text { Anaphalis javanica (B1.) } \\
\text { Boerl. }\end{array}$ & 2,701 & Clumped \\
\hline 3 & $\begin{array}{l}\text { Anaphalis longifolia (B1.) } \\
\text { D.C. }\end{array}$ & 7 & Clumped \\
\hline 4 & Chromolaena odorata L. & 3,397 & Clumped \\
\hline 5 & $\begin{array}{l}\text { Crassocephalum crepidioides } \\
\text { (Benth.) S.Moore }\end{array}$ & 7 & Clumped \\
\hline No & Scientific name & $\begin{array}{l}\text { Morista } \\
\text { Indeces }\end{array}$ & $\begin{array}{c}\text { Distribution } \\
\text { patterns }\end{array}$ \\
\hline 6 & $\begin{array}{l}\text { Dichrocephala } \\
\text { chrysanthemifolia (B1.) DC. }\end{array}$ & 3,5 & Clumped \\
\hline 7 & Emilia sonchifolia $\mathrm{L}$ & 3,266 & Clumped \\
\hline 8 & Erigeron karviskianus L. & 2,25 & Uniform \\
\hline 9 & Eupatorium riparium $R e g$. & 1,154 & Clumped \\
\hline 10 & Lactuca serriola $\mathrm{L}$. & -1 & Uniform \\
\hline 11 & Mikania micrantha $\mathrm{L}$. & 1,5 & Clumped \\
\hline 12 & Shoncus arvensis L. & -1 & Clumped \\
\hline 13 & Taraxacum offinale L. & 1,866 & Clumped \\
\hline 14 & Tithonia diversifolia $\mathrm{L}$. & 7 & Clumped \\
\hline 15 & Youngia japonica $\mathrm{L}$. & -1 & Uniform \\
\hline
\end{tabular}

According to the research and Morista Indices calculation, distribution patterns categorized as clumped patterns (I > 1) observed in species of : Anaphalis javanica (B1.) Boerl., Anaphalis longifolia (B1.) D.C., Chromolaena odorata L., Crassocephalum crepidioides (Benth) S.Moore, Emilia sonchifolia L., Erigeron karviskianus L., Mikania micrantha L., Dichrocephala chrysanthemifolia (Bl.) D.C., Adenostema hirsutum L., Teraxacum officinale L., and Tithonia diversifolia L. Uniform type of distribution patterns observed in species of Lactuca serriola L., Shoncus arvensis L. and Youngia japonica L. Almost $80 \%$ of asters species in Cemoro Kandang hiking trail have clumped patterns.

Potential analysis of Asteraceae Family as ecotourism attraction

The analysis have been done with interview and questionnare method which distributed to 86 respondences. The results of interview and questionnare implementation is shows below

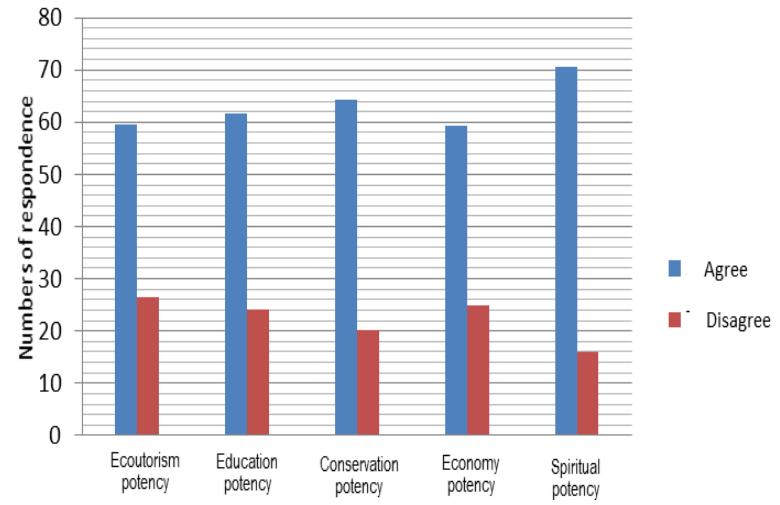

Fig. 2. Public Opinion About Local Wisdom Potential as Ecotourism Support on Cemoro Kandang

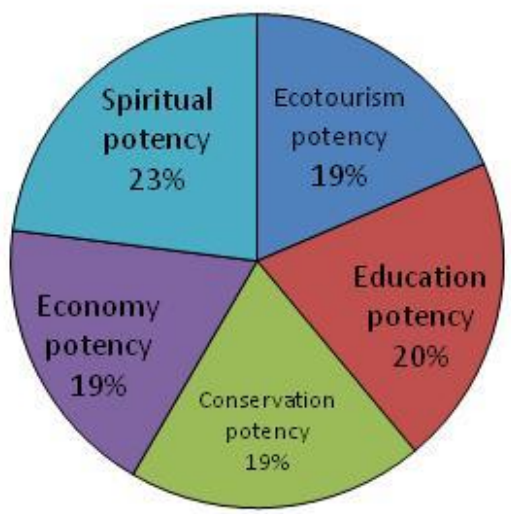

Fig. 3. Local Wisdom Potential Composition on Cemoro Kandang

\section{CONCLUSION AND SUGGESTIONS}

Research in Cemoro Kandang hiking trails discovered the diversity index of Asteraceae Family is 1, 14, classified as medium diversity. Highest abundance of Asteraceae mostly found in station V (altitude of $2600<\mathrm{A}<2800$ ). The numbers of individuals have been found is 41 , classified in 7 species including: Anaphalis javanica (B1.) Boerl., Taraxacum offinale L.,Youngia japonica L.,Tithonia diversifolia L., Eupatorium riparium Reg.,Emilia sonchifolia L., and Shoncus arvensis L. Lowest abundance observed in station II with 15 individual classified in only single species : Eupatorium riparium Reg. The distribution pattern of Asteraceae on Cemoro Kandang divided into two type: clumped and uniform. Dominant observed patterns is clumped (80\% from all invented species). The Asteraceae have potential as ecotourism attraction through their local wisdom values including natural tourism potency (19\%), education potency $(20 \%)$, conservation value $(19 \%)$, economic value $(19 \%)$ and spiritual value $(23 \%)$.

\section{ACKNOWLEDGMENT}

Authors would like to thank The Chief of Karanganyar Environmental Agency, Institution of Research and Communities Dedication of Sebelas Maret University, Anak Gunung Lawu (AGL) Community and other relevance institutions which give valuable contribution and facilities as 
effort to support this Cemoro Kandang ecotourism development research.

\section{REFERENCES}

[1] Fandeli, C. 2000. "Pengusahaan Ekowisata. Yogyakarta: Gadjah Mada University Press.Kayowuan, Y.A. 2014. Studi Kerusakan Edelweis (Anaphalis javanica) akibat Kegiatan Pendakian pada Jalur Pendakian Resmi Gunung Semeru - BBTNBTS”. Artikel Penelitian. Malang: Fakultas Kehutanan Institut Pertanian Malang.

[2] Maisyaroh, W. 2010. "Struktur Komunitas Tumbuhan Penutup Tanah di Taman Hutan Raya R Soerjo Cangar Malang," Jurnal Pembangunan dan Alam Lestari 1(1):1-9.

[3] Odum E.P, 1993, "Dasar-dasar Ekologi”, Yogyakarta: Gadjah Mada University Press.

[4] Subhashini S., and T.V. Poonguzhali, 2014, "Anatomicalprotocol for Botanical Diagnosis of a Time-Renowned Indian Herbal Sphaeranthus Amaranthoides Burm. F. (Asteraceae)," International Journal Of Recent Scientific Research 5(6):1050-1052.

[5] Van Steenis C.G.G.J., 1987, "Flora,” Jakarta: Pradnya Paramita.

[6] Van Steenis C.G.G.J., 2010, "Flora Pegunungan Jawa," Edisi Indonesia 2010, Diterjemahkan oleh: Jenny A. Kartawinata, Jakarta: Grafika Desa Putera 